\title{
Valence Quarks Polarization from COMPASS
}

A.Korzenev, for the COMPASS collaboration

Mainz University, Institute of Nuclear Physics, D-55099, Mainz, Germany

\begin{abstract}
A first evaluation of the polarized valence quark distribution $\Delta u_{v}(x)+\Delta d_{v}(x)$ from the COMPASS experiment (CERN/SPS) is presented. The data were collected by COMPASS in the years 2002-2004 using a $160 \mathrm{GeV}$ polarized muon beam scattered off a large polarized ${ }^{6} \mathrm{LiD}$ target and cover the range $1<Q^{2}<100 \mathrm{GeV}^{2}$ and $0.006<$ $x<0.7$. The analysis is based on the difference asymmetry, $A^{h^{+}-h^{-}}$, for hadrons of opposite charges, which gives a direct access to the polarization of valence quarks.
\end{abstract}

\section{Introduction}

Nowadays, there is growing of interest in semi-inclusive deep inelastic scattering experiments (SIDIS) with longitudinally polarized beams and targets as they provide an additional information on the spin structure of the nucleon compared to the inclusive DIS measurements. The SIDIS data allow to separate the valence and sea contributions to the nucleon spin.

Previous measurements of the valence quark helicity distributions were done by the SMC $[2,3]$ and the HERMES [4] collaborations. The SMC data cover a similar kinematic range as the COMPASS data, but with statistics which is an order of magnitude lower. HERMES has a high statistics data set and PID, thus it can disentangle all five quark helicity distributions. However the $x$-range is quite limited: $0.023<x<0.6$.

In the present analysis we use the so called difference asymmetry which is determined from the difference of cross sections of positive and negative hadrons $h^{+}$and $h^{-}$:

$$
A^{h^{+}-h^{-}}=\frac{\left(\sigma_{\uparrow \downarrow}^{h+}-\sigma_{\uparrow \downarrow}^{h-}\right)-\left(\sigma_{\uparrow \uparrow}^{h+}-\sigma_{\uparrow \uparrow}^{h-}\right)}{\left(\sigma_{\uparrow \downarrow}^{h+}-\sigma_{\uparrow \downarrow}^{h-}\right)+\left(\sigma_{\uparrow \uparrow}^{h+}-\sigma_{\uparrow \uparrow}^{h-}\right)} .
$$

Here arrows indicate the relative direction of the beam and target polarizations. The difference asymmetry approach was developed and used in SMC [5, 2]. Results obtained with this approach, as compared to the traditional single hadron approach $[3,4]$, are "cleaner" from the theoretical point of view because of the very weak sensitivity of $A^{h^{+}-h^{-}}$to uncertainties coming from fragmentation functions (FF). As it is shown in [5] FFs cancel out from $A^{h^{+}-h^{-}}$ in LO QCD. For the deuteron target the asymmetry is:

$$
A_{d}^{h^{+}-h^{-}} \equiv A_{d}^{\pi^{+}-\pi^{-}}=A_{d}^{K^{+}-K^{-}}=\frac{\Delta u_{v}+\Delta d_{v}}{u_{v}+d_{v}}, \quad \text { where } \quad \Delta q_{v} \equiv \Delta q-\Delta \bar{q} .
$$

The fact that kaons contribute to the asymmetry exactly like pions allows to avoid statistical losses due to hadron identification. Starting from NLO QCD the difference asymmetry depends also on FFs. However their effect is small [6].

The single hadron asymmetries $A^{h+}$ and $A^{h-}$ can be used to obtain $A^{h^{+}-h^{-}}$:

$$
A^{h^{+}-h^{-}}=\frac{1}{1-r}\left(A^{h+}-r A^{h-}\right), \quad \text { with } \quad r=\frac{\sigma_{\uparrow \downarrow}^{h-}+\sigma_{\uparrow \uparrow}^{h-}}{\sigma_{\uparrow \downarrow}^{h+}+\sigma_{\uparrow \uparrow}^{h+}}=\frac{N^{-}}{N^{+}} \cdot \frac{a^{+}}{a^{-}} .
$$

*Supported by the BMBF. On leave from JINR, Dubna, Russia. E-mail: korzenev@mail.cern.ch 

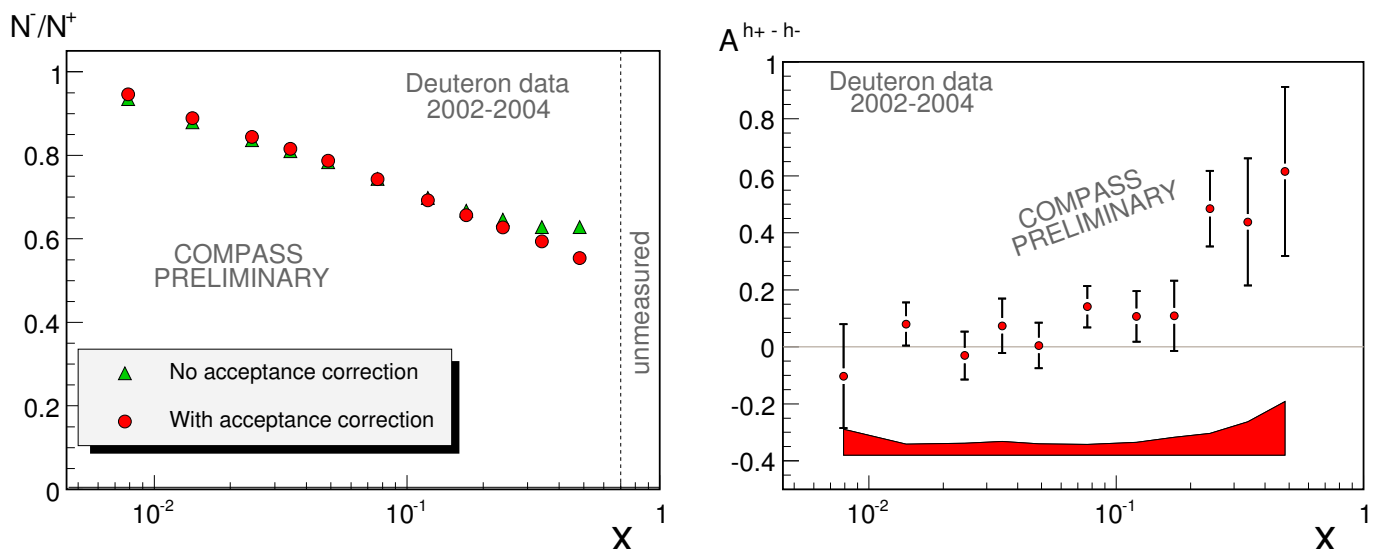

Figure 1: Left: The hadron number ratio $N^{-} / N^{+}$and the same ratio corrected for the acceptance which represents $\sigma^{h^{-}} / \sigma^{h^{+}}$. Right: Difference asymmetry $A^{h^{+}-h^{-}}$.

The ratio of cross sections for negative and positive hadrons, $r$, depends on the event kinematics and could, in principle, be measured in unpolarized experiments. In practice, it will be obtained from the hadron number ratio $N^{-} / N^{+}$corrected by the ratio of their acceptances $a^{-} / a^{+}$.

Since the deuteron is an isoscalar target we can not distinguish between up and down quarks. Nevertheless having measured the first moment of $\Delta u_{v}(x)+\Delta d_{v}(x)$ and combining its value with axial charges $a_{0}$ and $a_{8}$ the information about the symmetry of sea quark distributions can be extracted. Since $\Delta s+\Delta \bar{s}=\frac{1}{3}\left(a_{0}-a_{8}\right)$ one can show that

$$
\Delta \bar{u}+\Delta \bar{d}=(\Delta s+\Delta \bar{s})+\frac{1}{2}\left(a_{8}-\Gamma_{v}\right), \quad \text { where } \quad \Gamma_{v}=\int_{0}^{1}\left(\Delta u_{v}(x)+\Delta d_{v}(x)\right) d x .
$$

The $S U(3)_{f}$ symmetric sea $(\Delta \bar{u}=\Delta \bar{d}=\Delta s=\Delta \bar{s})$ will obviously lead to $\Gamma_{v}=a_{8}$. In contrast, if measurements give $\Gamma_{v}=a_{8}+2(\Delta s+\Delta \bar{s})$ it will point to a strong asymmetry for the first moments of light sea quarks $\Delta \bar{u}=-\Delta \bar{d}$.

\section{Extraction of the asymmetry}

In the analysis data collected during the years 2002-2004 have been used. We require for all events to have a reconstructed primary interaction vertex defined by the incoming and the scattered muons. The energy of the beam muon is constrained to be in the interval $140<E_{\mu}<180 \mathrm{GeV}$. To equalize fluxes through the two target cells it is required for the trajectory of the incoming muon to cross both cells. The kinematic region is defined by cuts on the photon virtuality $Q^{2}$ and the fractional energy $y$ transfered from the beam muon to the virtual photon. The requirement $Q^{2}>1 \mathrm{GeV}^{2}$ selects the region of DIS. The cut $y>0.1$ removes events which are problematic from reconstruction point of view due to a small energy transfer. The region which is the most affected by radiative corrections is eliminated with the cut $y<0.9$. At low $x$ (high $W$ ), where cross-sections of positive and negative hadrons are approximately equal, the statistical error of $A^{h^{+}-h^{-}}$increases drastically. Due to this 

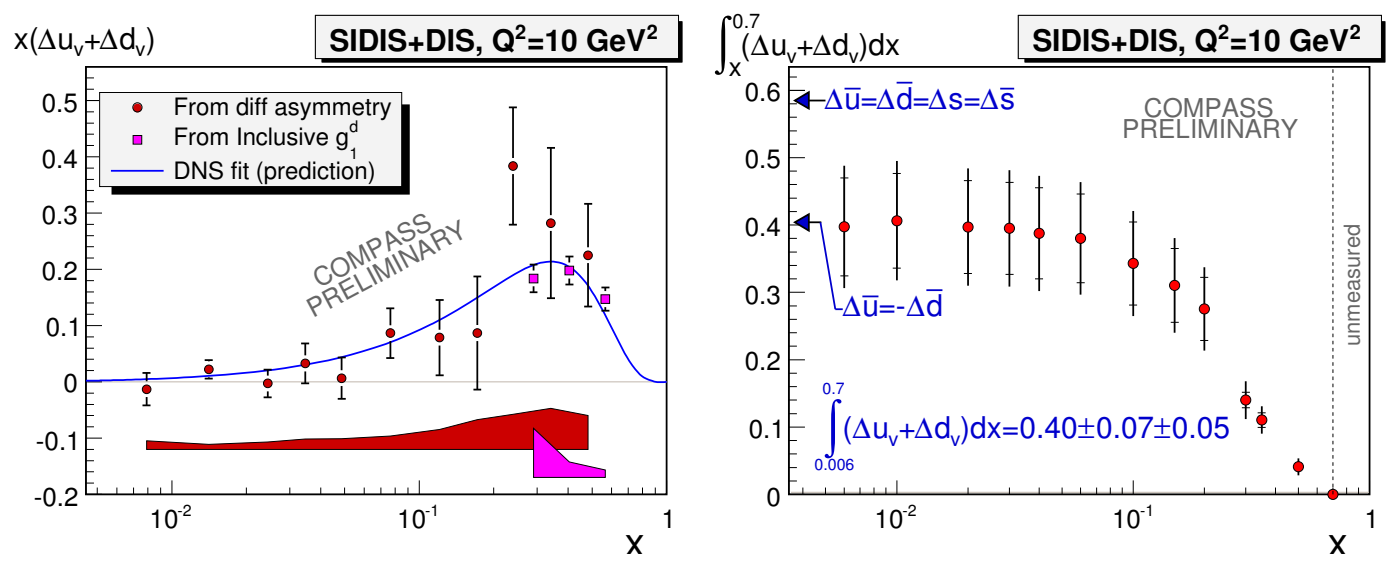

Figure 2: Left: Polarized valence quark distribution $x\left(\Delta u_{v}+\Delta d_{v}\right)$ evolved to $Q^{2}=10 \mathrm{GeV}^{2}$ according to the DNS fit at LO [8]. The line shows the prediction from the fit. Right: Corresponding integral of $\Delta u_{v}(x)+\Delta d_{v}(x)$ as the function of the low $x$ limit of integration.

reason we consider only $x>0.006$. For hadron tracks coming from the primary vertex the cut $z>0.2$ is applied to select the current fragmentation region. Hadron identification is not used. The resulting sample contains 30 and 25 millions of positive and negative hadrons, respectively.

The contributions to the systematic error from the target and beam polarizations, the dilution and depolarization factors amount to $8 \%$ of the asymmetry value when added in quadrature. The upper limit of the false asymmetry which could be generated by instabilities of the spectrometer components was evaluated as a fraction of statistical error: $\sigma_{\text {false }}<$ $0.5 \sigma_{\text {stat }}$. The asymmetry $A^{h^{+}-h^{-}}$with its statistical and systematic errors is shown in Fig. 1.

\section{Extraction of $\Delta u_{v}+\Delta d_{v}$ and its first moment}

The values of $\Delta u_{v}+\Delta d_{v}$ are obtained by multiplying $A^{h^{+}-h^{-}}$by the unpolarized valence distribution of MRST 2004 at LO [7]. Corrections for the deuteron D-state contribution and for the fact that the unpolarized parton distributions originates from $F_{2}$, in which $R=\sigma_{L} / \sigma_{T}$ was different from zero, are applied

$$
\Delta u_{v}+\Delta d_{v}=\frac{u_{v}+d_{v}}{\left(1+R\left(x, Q^{2}\right)\right)\left(1-1.5 \omega_{D}\right)} A^{h^{+}-h^{-}} .
$$

The evaluation of the first moment, $\Gamma_{v}$, requires the evolution of all $\Delta u_{v}(x)+\Delta d_{v}(x)$ points to a common $Q^{2}$. This is done by using the DNS parametrization in LO [8] which is based on the global QCD analysis of all DIS $g_{1}$ data prior to COMPASS as well as the SIDIS data from SMC and HERMES. The parametrization corresponding to KKP fragmentation functions was used. The resulting distribution at $Q^{2}=10 \mathrm{GeV}^{2}$ is shown in Fig. 2. A good agreement of the curve with the COMPASS points illustrates the consistency between the three experiments.

For $x>0.3$ the unpolarized sea contribution to $F_{2}$ practically vanishes. Due to positivity conditions $|\Delta q|<q$ the polarized sea contribution to the spin of the nucleon also can be 


\begin{tabular}{|l||c|c||c|c|r|c|}
\hline \multicolumn{1}{|c||}{$x$-range } & \multirow{2}{*}{$\begin{array}{c}Q^{2} \\
\mathrm{GeV}^{2}\end{array}$} & & \multicolumn{2}{c|}{$\Delta u_{v}+\Delta d_{v}$} & \multicolumn{2}{c|}{$\Delta \bar{u}+\Delta d$} \\
\hline \hline SMC 98 & & Value of the exper. & DNS & Value of the exper. & DNS \\
\hline HERMES 05 & $0.003-0.7$ & 10 & $0.26 \pm 0.21 \pm 0.11$ & 0.386 & $0.02 \pm 0.08 \pm 0.06$ & -0.009 \\
\hline \hline COMPASS & $0.006-0.7$ & 2.5 & $0.43 \pm 0.07 \pm 0.06$ & 0.363 & $-0.06 \pm 0.04 \pm 0.03$ & -0.005 \\
\hline
\end{tabular}

Table 1: Estimates of the first moments $\Delta u_{v}+\Delta d_{v}$ and $\Delta \bar{u}+\Delta \bar{d}$ from the SMC [3], HERMES [4], COMPASS data and also from the DNS fit at LO [8].

neglected. It allows to replace at LO Eq. (5) by

$$
\Delta u_{v}+\Delta d_{v}=\frac{36}{5} \frac{g_{1}^{d}\left(x, Q^{2}\right)}{\left(1-1.5 \omega_{D}\right)}-\left(2(\Delta \bar{u}+\Delta \bar{d})+\frac{2}{5}(\Delta s+\Delta \bar{s})\right)
$$

which gives a much more precise evaluation of $\Delta u_{v}+\Delta d_{v}$ at high $x$. In the calculation we omit the second term of the right side of this equation. However it is used to evaluate the systematic error. The values of $g_{1}^{d}$ from [9] were used. In total, we obtain

$$
\left.\Gamma_{v}(0.006<x<0.7)\right|_{\mathrm{Q}^{2}=10 \mathrm{GeV}^{2}}=0.40 \pm 0.07 \text { (stat.) } \pm 0.05 \text { (syst.), }
$$

which is $2 \sigma$ below the value corresponding to a flavor symmetric sea and very close to the value expected for $\Delta \bar{u}=-\Delta \bar{d}$ (see Eq. (4) where $\Delta s+\Delta \bar{s}$ is taken from [9]). The comparison with first moments obtained with results of SMC and HERMES can be found in Tab. 1.

As one can judge from Fig. 2 the integral is practically constant at low $x$. Thus the low $x$ contribution to $\Gamma_{v}$ is expected to be negligible. The contribution to $\Gamma_{v}$ for $x>0.7$ estimated with the LO DNS parametrization is 0.004 .

\section{Conclusion}

A first LO evaluation of the polarized valence quark distribution $\Delta u_{v}(x)+\Delta d_{v}(x)$ from the COMPASS deuteron data is presented. The data were collected by COMPASS in the years $2002-2004$ and cover the range $1<Q^{2}<100 \mathrm{GeV}^{2}$ and $0.006<x<0.7$. The analysis was based on the difference asymmetry approach. It leads to the first moment of $\Delta u_{v}+\Delta d_{v}$ : $0.40 \pm 0.07$ (stat.) \pm 0.05 (syst.) which favors the "asymmetric" light sea scenario $\Delta \bar{u}=-\Delta \bar{d}$ as compared to the "symmetric" one $\Delta \bar{u}=\Delta \bar{d}=\Delta s=\Delta \bar{s}$.

\section{References}

[1] Slides: http: //indico. cern. $\mathrm{ch} /$ contributionDisplay . py? contribId=144\&sessionId=4\&conf Id=9499

[2] (SMC) B. Adeva et al., Phys. Lett. B369 93 (1996).

[3] (SMC) B. Adeva et al., Phys. Lett. B420 180 (1998).

[4] (HERMES) A.Airapetian et al., Phys. Rev. D71 012003 (2005).

[5] L.L. Frankfurt et al., Phys. Lett. B230 141 (1989).

[6] A.N. Sissakian, O.Yu. Shevchenko and O.N. Ivanov, Phys. Rev. D73 094026 (2006).

[7] A.D.Martin, W.J.Stirling and R.S.Thorne, Phys. Lett. B636 259 (2006).

[8] D. de Florian, G.A.Navarro and R.Sassot, Phys. Rev. D71 094018 (2005).

[9] (COMPASS) V.Yu.Alexakhin et al., Phys. Lett. B647 8 (2007). 